\title{
Reflexiones acerca del sistema de pensiones
}

Andrés Suárez ${ }^{\dagger}$

Profesor de Ética Empresarial, Universidad Alberto Hurtado

\section{El malestar}

En 2016 se generalizó un malestar hacia el sistema de AFP que se hizo cada vez más grande y que finalmente tuvo su expresión más visible en masivas marchas públicas convocadas por críticos hacia este sistema. Por un lado, pareciera ocurrir que el actual sistema de pensiones al cual “la ley nos obligó" años atrás más que beneficiarnos nos habría estafado $y$, por el otro, el que las rentabilidades de las AFP hayan sido por largo tiempo "excesivas" constituiría un acto inmoral para la sociedad pues no redunda en un beneficio para el afiliado.

Se distinguen ciertos puntos críticos en los que el origen del malestar podría estar en la imposibilidad de comprender bien las características del sistema de AFP, entre éstas:

- La forma en que se generan rentas para las empresas y el cobro de los costos para el afiliado

- La (des)información de los afiliados sobre el desempeño de sus fondos y la débil educación financiera de los ciudadanos.

- El verdadero significado de que las utilidades hayan sido "excesivas" durante largos períodos

- La observación de una conducta monopólica permanente en la industria en diferentes períodos de tiempo, como si no hubiese competencia entre las empresas

- La identificación de cuál es la AFP líder - El dilema moral que plantea saber ya con certeza que viviremos muchos años más: una esperanza de vida de 85 años en promedio para 2050, contra los 71 años que se esperaban al momento de diseñarse el sistema de pensiones, ¿de verdad las AFP nos ven como una persona que vivirá 110 años, según las tablas de mortalidad que elaboran las entidades reguladoras del sistema?
Según cita la Comisión Bravo (2015), ya el programa de gobierno de Michelle Bachelet en 2013 había abordado las expectativas en la calidad de las pensiones: los años de trabajo no se condicen con la baja pensión recibida por los trabajadores. A su vez, éste consignaba "altas tasas de evasión y elusión de las cotizaciones entre los asalariados", asociadas a variables tales como el sector económico del afiliado, la región geográfica, género y oficio.

A su vez, podríamos preguntarles a los elaboradores del "programa", ies esta baja tasa de reemplazo -monto percibido como pensión versus el monto de los ingresos medios de trabajadorexpresión de una alta tasa de ahorro o es simplemente el resultado de que el ciudadano no supo cómo ahorrar lo suficiente?

Por otro lado, el movimiento"No más AFP", por ejemplo, articula sus críticas en cinco puntos claves: (1) unas pensiones bajas que no se condicen con un "sistema exitoso", (2) una concentración del capital en pocos empresarios y familias, (3) lobby político y corrupción, para que las principales empresas de la industria puedan mantener el "monopolio previsional", (4) falta de competencia del sistema, aludiendo a la establecimiento de un "oligopolio" $y_{\text {, }}(5)$ una reforma previsional "fraudulenta" -la de 2008- que concibe "la seguridad social en un negocio lucrativo para las AFP y empresas vinculadas" (sic). ¿Qué tantas razones tendrían estos argumentos? El mero reclamo invocando un sentir ciudadano no es una condición sine qua non de contar con los suficientes análisis para calificar a una industria de corrupta ni de situar al lucro como un valor negativo per se.

\section{La difícil comprensión}

Las AFP comunican a sus afiliados mes a mes el desempeño de los fondos. Así también, de las caídas de estos fondos y aún de la potencial recuperación parcial del valor de la cuota, como ocurrió en 2016. Es difícil para el afiliado comprender correctamente el vaivén de 
sus ahorros para la vejez. Al respecto, Vial y Melguizo (2009), analizando diversos sistemas de fondos de pensiones, expresaban:

"Una crítica común al sistema privado de pensiones basado en cuentas individuales y libre afiliación a la AFP (esto es, basado en la competencia) es que los cargos son muy altos y terminan constándole mucho a los afiliados. Este tema es muy complejo, pues los costos varían dependiendo de la etapa del ciclo de vida de cada afiliado, así como de cuán maduro esté el sistema. Ello es bastante confuso por dos hechos. Primero, las contribuciones a los fondos de pensiones se expresan generalmente como una fracción de los ingresos, donde las comisiones de los mercados financieros se calculan como fracción de los activos bajo administración. Segundo, las comisiones pagan diferentes cosas en diferentes países. En muchos casos (Chile es uno de ellos) esto incluye seguros para la cobertura de sobrevivientes y seguros de discapacidad (aproximadamente la mitad de los cargos en Chile van a cubrir este seguro) (Vial y Melguizo, 2009:17)

Cómo se puede ya intuir, la primera gran barrera es la comprensión del sistema, y nuestra educación e información es un punto débil. La Comisión Bravo (2015), iniciativa gubernamental para el análisis de sistema de pensiones, concluye que "existe un escaso conocimiento de las personas" sobre el sistema, "poco conocimiento sobre cómo se calculan las pensiones y cuál es la tasa de contribución" y "pocos espacios de educación". Si los espacios son pocos, nuestra educación modesta y también nuestro interés, ¿es nuestra pasividad parte de la fuente de la desinformación?, ¿esta- mos preparados para evaluar y opinar si no estamos educados sobre cómo funcionan el sistema que administra nuestros fondos para la vejez?

Vial (2013) describe cuál es el fondo de la cuestión: "a nivel de cotizantes de ingresos medios y altos hay problemas de insuficiencia de ahorros para tener tasas de reemplazo elevadas".

Entre los países de la OCDE la tasa de reemplazo para el trabajador con salario promedio, Chile cuenta con la segunda tasa de reemplazo más baja, con un $38 \%$, siendo la más baja la de México, con un $26 \%$ (OCDE, 2016). Es decir, es a tal porcentaje que se reducirían mis ingresos al momento de jubilar. Un gerente de riesgo decía en una conversación, "lo que la gente no entiende es que deben ahorrar más". Pero, ¿es sólo una cuestión de sentido común? Tal vez no, pues la capacidad de ahorro con un coeficiente de Gini tan desigual en nuestro país deja la posibilidad de ahorrar a segmentos muy específicos de la población local. Ahorrar significa vivir con menos hoy, ¿y estamos dispuestos?, ¿cuántos pueden sacrificar el consumo presente sin dañar cualitativamente su calidad de vida?, ¿cuántos pueden y desean hacer el cálculo apropiado?, ¿desde cuándo las AFP nos acompañan de cerca en el desempeño de nuestros fondos?

\section{Un pasado monopólico con utilidades extranormales}

Diversos estudios del análisis del desempeño de la industria (Valdés y Marinovic, 2005; Ravizza, 2012; López, 2016) revela utilidades extra normales, y este punto es uno de los que más exacerba a la opinión pública: ¿por qué si las empresas ganan tanto es que las pensiones serían tan bajas? Esta característica de utilidad extra normal es más propia a la de un monopolio natural sin regulación con economías de escala decrecientes y altas barreras a la entrada, y menos a la de un mercado potencialmente competitivo donde participan varias empresas. Pero claro, una cuestión es el desempeño de la industria y, otro, el de los fondos ahorrados por el afiliado. Si la utilidad extranormal no es ilegal, ¿deben los afiliados tolerarlas?, ¿no nos queda otra?

Hoy existen seis AFP y desde 1981 se pueden contabilizar 34. Según la información que nos proporciona la Superintendencia de Pensiones (Noviembre de 2016) el valor de los fondos de pensiones para 2015 está concentrado en cuatro AFP, a saber, Provida (27\%), Hábitat (26\%), Cuprum (21\%) y Capital (20\%). La participación de las dos AFP restantes es modesta, siendo de un 3\% para Planvital y de un $2 \%$ para Modelo. Por otro lado, estas AFP más pequeñas son las que menos comisión cobran a sus afiliados. ¿Es que el ciudadano está tan desinformado y tan pasivo que como actor económico que su falta de información (o educación) no promovido la competencia cambiándose hacia aquellas AFP que menos comisión le cobran según sus tramos de ingreso?

A nivel agregado, nuestros fondos se invierten principalmente en el extranjero en Cuotas de Fondos Mutuos y Acciones y en Instrumentos de Deuda, por un lado, y en Bonos de la Tesorería General de la República. El mayor porcentaje de nuestros fondos se invierten en el sector extranjero $(44 \%)^{1}$ y el Sector Estatal $(23 \%)^{2}$.

Le siguen el sector financiero (18\%) y el sector empresas $(15 \%)^{3}$. (Superintendencia de Pensiones, 2016). ¿Puede el ciudadano comprender y verificar que una AFP le informe, de acuerdo a

(1) El sector extranjero está conformado por Cuotas de Fondos Mutuos y Acciones, Inversión indirecta en el extranjero, Instrumentos de Deuda y Forwards. Este sector, en diciembre de 2015, está representado en un 33\% por Cuotas de Fondos Mutuos y Acciones y en $11 \%$ por Instrumentos de deuda.

(2) El sector estatal está conformado por instrumentos del Banco Central de Chile, de la Tesorería General de la República, Bonos de Reconocimiento e instrumentos del MINVIU, en donde sobresalen los de la Tesorería, con un 17\% (diciembre de 2015). El sector financiero está conformado por Letras Hipotecarias, Depósitos a Plazo, Bonos de Instituciones Financieras, acciones de Instituciones Financieras y forwards. Los Depósitos (7,1\%) y Bonos (9,6\%) son los principales instrumentos.

(3) En el sector empresas está conformado por Acciones, Bonos y Cuotas de Fondos de Inversión. Las acciones (6,7\%) y los Bonos (6,4\%) son los instrumentos por excelencia. 
la estructura de cartera, que las elecciones en Estados Unidos y la nominación de Donald Trump están por detrás del mal desempeño y los resultados que obtienen sus fondos?

Las rentabilidades excesivas parecen el sino de la industria. López (2016) señala que "la rentabilidad sobre patrimonio promedio de las AFP en el periodo $1991-2015$ fue $26,4 \%$, cifra que supera en $66 \%$ al $16 \%$ alcanzado por la banca en el mismo periodo". La misma investigación expone que dicha rentabilidad sobre patrimonio fue 4,7 veces superior al 5,3\% justificable por su exposición al riesgo de mercado. Por otro lado, AFP Capital y Provida cobran comisiones que son 3,5 y 3,8 veces superiores a las de la AFP más barata de la industria (Planvital), sin que estas diferencias se justifi-

Valdés y Marinovic (2005), al examinar el desempeño de la industria en el período 1993-2003, ponían ya sobre el tapete la alta rentabilidad observada, indicando que desde "es inusual encontrar industrias de gran tamaño que, en su conjunto, obtengan una rentabilidad sobre activos de entre $35 \%$ y $68 \%$ anual, que superan ampliamente a la rentabilidad normal, por períodos de seis años, como ocurrió en 1998-2003, y que al mismo tiempo no se observe entrada (desde 1996)" (Valdés y Marinovic, 2005:41).

Por otro lado, Ravizza (2012) analizó la conducta competitiva en la industria de las AFP en Chile para el periodo 2003-2009, y la reforma al sistema de pensiones llevada a cabo en 2008. En su estudio concluye que sus resultados "permitirían dar una justificación empírica para las conjeturas de una baja competencia en la industria que originaron las reformas pro-competitivas de 2008" (Ravizza, 2012:36). La autora afirma que el análisis dinámico del comportamiento competitivo en el período de estudio permite indicar

quen por diferencias en sus rentabilidades esperadas (Lara y otros, 2016). En efecto, si bien el financiamiento de las AFP proviene del cobro de comisiones a sus afiliados, dicho monto se añade al de nuestra cotización obligatoria del $10 \%$ sobre nuestra renta imponible. Según la Superintendencia

\section{Tabla 1}

Estructura de comisiones por depósito de cotizaciones, noviembre de 2016

\begin{tabular}{ll} 
AFP & $\begin{array}{l}\text { \% de la remuneración o } \\
\text { renta imponible }\end{array}$ \\
\hline CAPITAL & 1.44 \\
\hline CUPRUM & 1.48 \\
\hline HABITAT & 1.27 \\
\hline MODELO & 0.77 \\
\hline PLANVITAL & 0.41 \\
\hline PROVIDA & 1.54 \\
\hline
\end{tabular}

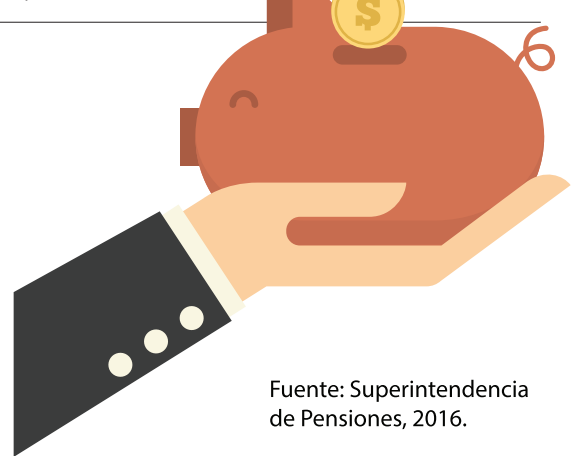

la existencia de conducta monopólica para la industria y que los resultados se relacionarían con la hipótesis en la cual las firmas podrían haber estado en una coordinación implícita para evitar una guerra comercial entre ellos, auto restringiéndose a sí mismas en el ejercicio de su poder de mercado. A partir de lo anterior la autora asevera que, "de este modo, esta conjetura permitiría inferir que a medida que se hacía más probable la intervención al mercado, las firmas podrían haber relajado sus restricciones autoimpuestas y ello podría justificar el alza en las comisiones y en los ingresos totales" (Ravizza, 2012:35-36).

¿Es que estamos asignando bien los recursos de nuestra economía? La alta utilidad de las empresas de la industria, debería promover el ingreso de nuevos competidores. La rentabilidad observada por un extenso periodo de tiempo es superior a la rentabilidad que se justifica por el nivel de riesgo del negocio en el caso en estudio. Es como si no hubiese ocurrido jamás que nuevos ac- de Pensiones, en noviembre de 2016 las comisiones más bajas pertenecían a Planvital $(0.41 \%)$ y Modelo $(0,77 \%)$. Las cuatro primeras compañías concentran, no sólo el valor de los fondos sino que cobran hasta el doble de lo que cobra una AFP más barata, según podemos observar en la Tabla № 1. de Pensiones, 2016

tores hayan tenido incentivos para ingresar, tal como señala la teoría económica. La última compañía en ingresar a la industria fue AFP Modelo en 2010, que no provino de ninguna fusión anterior. Así, se cumple lo mencionado por López, que "cuando un grupo de empresas tiene la capacidad de generar rentabilidades elevadas por un periodo sostenido de tiempo, generalmente se considera como un síntoma de falta de competitividad [...y] esta falta de competitividad distorsiona la asignación de recursos de la economía" (López, 2016:103). Es decir, los excedentes quedan en el productor y mucho menos en el consumidor: se produce ineficiencia social.

¿Es moral ser ineficiente si es legal? En nuestro país, la utilidad extra normal de las empresas de la industria es absolutamente legal.

\section{La AFP líder}

Lara y otros (2016) analizan si existe algún líder en rentabilidad, examinando el 
sistema de AFP en tres períodos: 19812015 (desde la creación del sistema de capitalización individual), 2002-2015 (desde la creación de los multifondos) y 2010-2015 (desde la implementación de la reforma al sistema de pensiones de 2008). Habiendo considerado variables tales como la distribución de ingreso imponible, saldo acumulado y distribución de los cotizantes en los diferentes fondos a diciembre de 2015 el estudio estimó cuál AFP deberían escoger los afiliados. Al respecto, los autores señalan que si las rentabilidades históricas de los fondos de pensiones se repitieran en el futuro "un $81 \%$ de los afiliados debería optar por las dos AFP más baratas de la industria", específicamente, que" $74 \%$ de ellos debería optar por la más barata (Planvital) y $7 \%$ por la segunda más barata (Modelo)" (Lara y otros, 2016). $Y$ es este tipo de información, cualitativamente de calidad e independiente, con la que hasta hace poco no contábamos: saber cuál AFP me conviene más.

¿Y qué dicen las mismas AFP de sí mismas? Hasta hace poco, diversas AFP decían ser "la № 1". Sin embargo, la primera información presentada en los sitios web de cada una es algo es algo diferente al finalizar el año 2016. El día 4 de diciembre de 2016 AFP Cuprum enunciaba en su sitio web que es la "№1 en Experiencia de Servicio" y "Tercer año consecutivo reconocidos como la mejor AFP". Mientras, AFP Habitat hace un llamado: "Aprovecha al máximo el beneficio tributario de tus productos voluntarios" $y$ en el link cambiate.afphabitat.cl promocionado por Google afirma que "sólo Habitat es $n^{\circ} 1$ en rentabilidad en todos los fondos a largo plazo y además $\mathrm{N}^{\circ} 1$ en APV". Por su parte, AFP Planvital lleva a cabo una afirmación: "La comisión más baja de todas las AFP: $0,41 \%$ ". Por su parte, AFP Modelo elabora una pregunta: “'Sabías que en AFP Modelo obtienes una alta rentabilidad y una baja comisión?". En tanto, Provida hace una invitación: "Te invitamos a aclarar las dudas sobre tu pensión y el funcionamiento del sistema". Capital, por su parte, en el link "las cosas por su nombre" ofrece responder las dudas sobre el sistema de pensiones. Ahora, todas las AFP están altamente interesadas en educarnos. ¿Tenían esta misma preocupación años anteriores, o es mera respuesta al escrutinio y el reclamo social? Que no se quejen las empresas de la industria si ha roto la confianza: este valor puede perderse muy rápidamente. Reconstruirlo, tomará años.

\section{Viviremos más: La extensión de la esperanza de vida}

Saber que viviremos más cada vez trae consigo determinar si alcanzarán nuestros ahorros para una pensión digna, quién solventará la parte que falte, dada la tasa de reemplazo, o saber por qué se construyen tablas de mortalidad con un margen de vida tan alto: ¿qué tan cierto es que voy a llegar a vivir 110 años?

Casi como corolario, podemos plantearnos, además, cómo hacer sostenible un sistema de ahorro para la vida futura con un escenario diferente al del presente. Si se acaban nuestros fondos se activan los seguros. El progresivo estancamiento de la población, ya presentado por Joaquín Vial desde el Banco Central en 2013 al comentar los retos de la longevidad en el documento "Desafíos de la transición demográfica para el sistema de pensiones de Chile" (Vial, 2013) planteaba ya algunos puntos críticos a analizar frente a la insuficiencia de ahorros para tener tasas de reemplazo elevadas, la irregularidad en las cotizaciones, la baja edad de jubilación de las mujeres y el aumento en la longevidad son los factores que más influyen. La primera línea de defensa para cubrir esa deficiencia, según el economista, son los ajustes en la edad de jubilación y los planes de ahorro voluntario. "El envejecimiento de la población -afirma- plantea desafíos al sistema, principalmente operacionales por el aumento de solicitudes de pensión y la necesidad de ampliar asesoría al jubilar". Asimismo, lo anterior precisaría "gestionar los riesgos asociados a 'sorpresas' en la esperanza de vida al jubilar". Y según su análisis, "la mejor solución pasa por reforzar el mercado de rentas vitalicias, mejorando su regulación, desarrollando la modalidad de renta temporal e introduciendo bonos de longevidad emitidos por el Estado" (Vial, 2013).

En efecto, vivimos cada vez más: en 2050 nuestra esperanza de vida al nacer (EVN) será de 85 años promedio. Si en 1953 las personas en Chile tenían una esperanza EVN de 54,8 años, para 2013 esta edad aumentó a 79,8. En sólo 60 años las personas aumentaron su EVN en un $40 \%$ (Larraín, 2016). Para el período 2010-2015 la EVN fue de 76,5 años para los hombres y de 81,7 para las mujeres; y para el siguiente quinquenio 2015 -2020 se estima que la EVN para los hombres será de 77,4 años y para las mujeres de 82,2 años (INE, 2015). Para los siguientes periodos la CEPAL ha producido información al respecto sobre Chile, que se ha condensado en la Figura 2, expresada en quinquenios.

Al respecto, podríamos afirmar, por una mala comprensión y educación en el sistema de pensiones, que son las empresas de la industria de las AFP las que estiman una edad de 110 años como tasa de mortalidad, pero no es así. Las tablas de mortalidad son elaboradas por la Superintendencia de Valores y Seguros y la Superintendencia de Pensiones. "Se usan tablas hasta 110 años porque hay un grupo pequeño de chilenos que llegan a esa edad y el instrumento de cálculo de pensión debe ser útil también para los longevos", enuncia la Asociación de AFP. En efecto, dada esta nueva ola de información surgida este 2016, la misma asociación enseña: "Es incorrecto interpretar que la pensión se calcule 
suponiendo que un jubilado vivirá con certeza hasta los 110 años, lo que hacen las tablas es estimar a una edad " $x$ ", la probabilidad de estar vivo a una edad futura " $x+n$ ". Este instrumento asigna -según la entidad- una probabilidad muy baja de alcanzar la edad de 110 años. La actual tabla en su versión inicial proyecta lo siguiente: de 100.000 hombres chilenos vivos a la edad de 20 años, alcanzarán la edad de 110 años sólo 17,15 hombres en promedio.

\section{Un juicio ético}

¿Qué valores están detrás del malestar? Desde el punto de vista moral hay dos valores en juego: la confianza, o la falta de ella, y el conocimiento de la verdad, o su desconocimiento.

Detrás de un modelo exitoso imitado por números países, en nuestro país llegamos a pensar qué tendría de exitoso este modelo si contrasta con los comunicados de las AFP y los mails a los cotizantes cuando le informan de la caída de los fondos. Lo natural es que el ciudadano se pregunte: ¿Cómo es esto posible, dadas las utilidades extra normales de la industria? En 1999 el think tank libertario norteamericano Tha Cato Institute, celebraba los 18 años de un modelo chileno de pensiones exitoso y aducía que las críticas que se le hacían a éste eran sólo"desinformación" (Rodríguez, 2009). Más recientemente, el 3 de agosto de 2016, el mismo ex ministro José Piñera, impulsor del modelo en plena dictadura e investigador del mismo centro de pensamiento, lo defendió sin temor. Adujo que los medios de comunicación falseaban la información periodística y que los gobiernos de la coalición de centro izquierda e izquierda posteriores a Pinochet confundían a la población pues hoy lo critican pero las políticas públicas [del período democrático] lo han consolidado. Leyó cuatro veces en la entrevista televisiva la carta enviada

\section{Figura 2}

Esperanza de vida al nacer, en quinquenios.

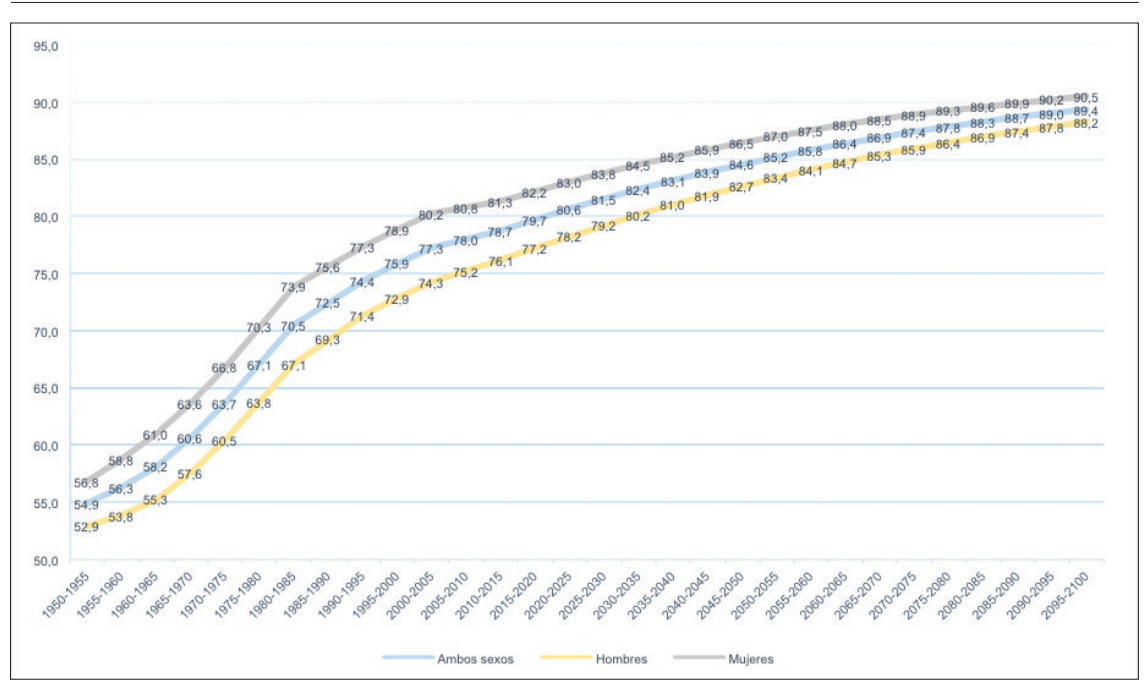

Fuente: CELADE - División de Población de la CEPAL. Revisión 2015

a los cotizantes de AFP Habitat, en la cual "Ios hombres que han cotizado por más de 30 años reciben una pensión promedio de $\$ 650.000 "$ (sic). Ahora bien, la Unidad de Data de El Mostrador, el 1 de julio de 2016 había calculado que el monto promedio pagado por AFP se estructuraba de la siguiente manera:

Tabla 2

Monto Promedio de pensiones en UF y Pesos

\begin{tabular}{ccc} 
Tipo de Pensión & $\begin{array}{c}\text { Monto Promedio de pensiones } \\
\text { por AFP en U.F en 2015 }\end{array}$ & $\begin{array}{c}\text { Monto en pesos según valor de } \\
\text { la UF de 1 de diciembre de 2016 }\end{array}$ \\
\hline Vejez & 7,34 & $\$ 193.154$ \\
\hline Invalidez Total & 8,66 & $\$ 227.890$ \\
\hline Vejez Anticipada & 11,7 & $\$ 307.889$ \\
\hline Total & 7,96 & $\$ 209.470$ \\
\hline
\end{tabular}

Fuente: Unidad de Data del Monstrador (2016) y SII

Escuchada una información, la de Piñera, y leídos los datos de El Mostrador, ¿quién nos está diciendo la verdad? O bien, ¿es que nos proporcionan una información sesgada, aprovechando nuestra anomia? El desafío para las empresas es su responsabilidad social respecto a "vender verdad". Los ciudadanos, si se han sentido engañados, en parte se debe a haber creído que las AFP eran un buen negocio para ellos también, pero hoy tienen la impresión de que sólo las empresas se han beneficiado del buen negocio, y esto es legal. $Y$ legal no tiene por qué ser moral.

Las grandes AFP han cambiado su metódico discurso mediático de ser «la número 1» matizando la información convenientemente para ellas. Las marchas, los cuestionamientos han demandado más verdad: los ciudadanos quieren saber cuáles son las ganancias, por qué son excesivas, y por qué los cotizantes no pueden captar esas rentabilidades de la AFP en los montos de sus ahorros. Este conocimiento de la verdad se enfrenta, a su vez, al nivel de la educación financiera con el que cuenta el ciudadano de a pie, por un lado, y la disponibilidad de información financiera, por el otro. Y si las personas no logran saber realmente cuál es su situación de sus ahorros o simplemente no leen 
sus cartolas, ¿es dable pensar que tomen decisiones racionales pensando en incrementar voluntariamente sus ahorros?

¿Seremos capaces de comprender no sólo la estructura específica de los fondos de inversión, sino también la estructura de las comisiones, el informe sobre desempeño de fondos y sumarlo a nuestra propia esperanza de vida para comprender que debemos ahorrar voluntariamente más? De acuerdo a la estructura de ingresos de las familias chilenas, estando entre los países más desiguales de la región latinoamericana, ¿están éstas en pie para ahorrar más?

Algunas voces alzan la voz diciendo que la propuesta de AFP estatal no sería el mejor camino, pues no es crucial quién desempeña el papel de administrador, sino, tomar la decisión de ahorrar más o, bien, de elevar los impuestos.

Las altas rentabilidades de las AFP no tienen un carácter inmoral. Es inmoral saber tan poco del funcionamiento del sistema de pensiones, y en parte es nuestra culpa como afiliados y ciudadanos. Nosotros tampoco nos hemos esforzado demasiado en comprender que el sistema tiene diferentes actores: AFP, bancos y compañías de seguros, el estado y nosotros. Todos somos parte del sistema, y hemos dejado que opere bajo una conducta monopólica sin competencia, sin estímulos para nuestro beneficio como afiliado.

\section{Bibliografía}

CEPAL (2016) Estimaciones y proyecciones de población a largo plazo 1950-2100. América Latina - Revisión 2015. Disponible en: http://www.cepal. org/es/estimaciones-proyeccionespoblacion-largo-plazo-1950-2100, última visita 30 de noviembre de 2016. Comisión Bravo (2015) Informe Final. Comisión Asesora Presidencial sobre el Sistema de Pensiones. Santiago, Septiembre de 2015. Disponible en: https://prensa.presidencia.cl/lficontent/otras/informes-comisiones/ InformePensiones.pdf, última visita 30 de noviembre de 2016.

Instituto Nacional de Estadísticas, INE (2015) Enfoque Estadístico Julio 2015. Disponible en www.ine.cl, última visita 30 de noviembre de 2016.

Lara, Daniel, Fernando López y Andrés Morgado (2016) Fondos de Pensiones: ¿Existe un líder en rentabilidad? Documento de Investigación I-315. Facultad de Economía y Negocios, Universidad Alberto Hurtado. Disponible en http://fen.uahurtado.cl/ wp-content/uploads/2015/05/201607-27-WP-Rentabilidad-FP.pdf, última visita 30 de noviembre de 2016.

Larraín, Guillermo (2016) “Un Sistema de Seguro para la Cuarta Edad". Con la colaboración de Simón Ballesteros y Sebastián García. Presentación para el II Seminario de Finanzas y Ética. Universidad Alberto Hurtado, 2 de Noviembre de 2016.

López, Fernando (2016) Industria de AFP Chilena: ¿Cuánto gana y cuánto debería ganar? Revista de Análisis Económico, Vol. 31, No 2, pp. 101114 (Octubre 2016). Disponible en: http://www.rae-ear.org/index.php/ rae/article/view/499/585, última visita 30 de noviembre de 2016.

López, Fernando (2016) Pensiones: Descontentos, Aspiracionesy Realidades. Observatorio Económico №116. Agosto de 2016. Disponible en http://fen. uahurtado.cl/publicaciones/oe/, última visita 30 de noviembre de 2016. Organización para el Cooperación y el Desarrollo Económico, OCDE (2016) Estudio de la OCDE sobre los sistemas de pensiones: México. Disponible en https://www.gob.mx/cms/uploads/ attachment/file/61968/sistema_de pensiones_2016.pdf, última visita 30 de noviembre de 2016.

Piñera, José (2016) Entrevista programa“EI Informante".Disponibleen https://www. youtube.com/watch?v=J-gD_B_hlxE, última visita 30 de noviembre de 2016.
Ravizza, Catalina (2012) Análisis de la Competencia en la Industria de las AFP en Chile: Enfoque NoEstructural. Tesis de grado Magister en Economía. Instituto de Economía, Pontificia Universidad Católica de Chile. Disponible en: http://economia. uc.cl/wp-content/uploads/2015/07/ tesis_cravizza.pdf , última visita 30 de noviembre de 2016.

Rodríguez, L. Jacobo (1999) 18 Years of Private Pensions in Chile. The Cato Institute. Disponible en https://www. cato.org/publications/commentary/18years-private-pensions-chile, última visita 30 de noviembre de 2016.

Superintendencia de Pensiones. Centro de Estadísticas de la Superintendencia de Pensiones. http://www.safp.cl/ safpstats/stats/

Unidad de Data de El Mostrador (2016) Chile con las pensiones más bajas de países OCDE. 1 de julio de 2016. Disponible en http://www.elmostrador. cl/noticias/pais/2016/07/01/chile-conlas-pensiones-mas-bajas-de-paisesocde/, última visita 30 de noviembre de 2016.

Valdés Salvador y Iván Marinovic (2005) Contabilidad Regulatoria: Las AFP Chilenas, 1993-2003. Instituto de Economía, Pontificia Universidad Católica de Chile. Documento de Trabajo № 279.

Vial, Joaquín (2013) Desafíos de la Transición Demográfica para el Sistema de Pensiones de Chile. Banco Central de Chile. Disponible en http://www.bcentral.cl, última visita 30 de noviembre de 2016.

Vial, Joaquín y Angel Melguizo (2008) Moving from pay-as-you-go to privately managed individual pension accounts: What have we learned after 25 years of the Chilean pension reform. Pensions: An International Journal, February 2009, Volume 14, Issue 1, pp 14-27. Disponible en http://link.springer. com/article/10.1057/pm.2008.32, última visita 30 de noviembre de 2016 . 